\title{
Mitogen activated protein kinases blockade improves lipopolysaccharide-induced ileal motor disturbances
}

\author{
Sergio Gonzalo, Laura Grasa, Ligia Verónica Hernández, María Pilar Arruebo, Miguel Ángel Plaza \\ and María Divina Murillo
}

Department of Pharmacology and Physiology. Unit of Physiology. Facultad de Veterinaria.

Universidad de Zaragoza. Zaragoza, Spain

\begin{abstract}
Background: several diseases such as sepsis can affect the ileum. Lipopolysaccharide (LPS), an endotoxin present in the cell wall of gram negative bacteria, is a causative agent of sepsis.

Objectives: the aims of this study were: a) to investigate the role of mitogen activated protein kinases (MAPKs) in the effect of LPS on the acetylcholine-induced contractions of rabbit ileum; and b) to study the localization of MAPKs in the ileum.

Material and methods: ileal contractility was studied in an organ bath and MAPKs were localized by immunohistochemistry.

Results: acetylcholine-induced contractions decreased with LPS SB203580, SP600125 and U0126 blocked the effect of LPS on the acetylcholine-induced contractions. Phosphorylated p38 and ERK were detected in neurons of myenteric plexus and phosphorylated p38 and JNK in smooth muscle cells of ileum.

Conclusion: we can suggest that p38, JNK, and ERK MAPKs are involved in the mechanism of action of LPS in the ileum.
\end{abstract}

Key words: Intestinal contractility. LPS. p38. ERK. JNK. Myenteric plexus. Smooth muscle.

\section{INTRODUCTION}

Gastrointestinal stasis is a common complication of sepsis $(1,2)$. Lipopolysaccharide (LPS), an endotoxin present in the cell wall of gram negative bacteria, is widely used to reproduce sepsis symptoms. For example, LPS administration results in a decrease in contractility of ileal longitudinal smooth muscle (3).

This work was presented, in part, at the "LXVII Congreso Anual de la Sociedad Española de Patología Digestiva (SEPD), Sitges-Spain June 2008”

Received: 22-11-2011

Accepted: 03-03-2012

Correspondence: María Divina Murillo. Department of Pharmacolology and Physiology. Unit of Physilogy. Facultad de Veterinaria. Universidad de Zaragoza. C/ Miguel Servet, 177. 50013 Zaragoza, Spain

e-mail: dmurillo@unizar.es
Gonzalo S, Grasa L, Hernández LV, Arruebo MP, Plaza MA and Murillo MD. Mitogen activated protein kinases blockade improves lipopolysaccharide-induced ileal motor disturbances. Rev Esp Enferm Dig 2012; 104: 305-309.

Mitogen activated protein kinases (MAPKs) are a family of protein kinases involved in many cellular programs such as cell proliferation, cell differentiation and cell death and it has been observed an abnormal activation of MAPKs in pathological circumstances such as sepsis. The most important groups of MAPKs are the p38, c-Jun-N-terminal kinase (JNK1/2) and extracellular signal-regulated kinase (ERK1/2) MAPK(4).

The aims of this study were: (1) to investigate the role of MAPKs in the effect of LPS on the acetylcholine-induced contractions of rabbit ileum and (2) to study the localization of MAPKs in the ileum.

\section{MATERIALS AND METHODS}

\section{Drugs and solutions}

Acetylcholine (ACh) and lipopolysaccharide (LPS, from Escherichia coli serotype 0111:B4) were purchased from Sigma (Madrid, Spain). 4-[5-(4-Fluorophenyl)-2-[4-(methylsulphonyl)phenyl]-1H-imidazol-4-yl]pyridinehydrochloride (SB-203580), anthra[1-9-cd]pyrazol-6(2H)-one (SP-600125), and 1,4-diamino-2,3-dicyano-1,4-bis[2-aminophenylthio]butadiene (U-0126) were acquired from Tocris (Madrid, Spain).

\section{Animals}

All experimental protocols were approved by the Ethics Committee of the University of Zaragoza (Spain). Male New Zealand rabbits weighing $2-2.25 \mathrm{~kg}$ were kept with standard rabbit fodder and free access to water. 


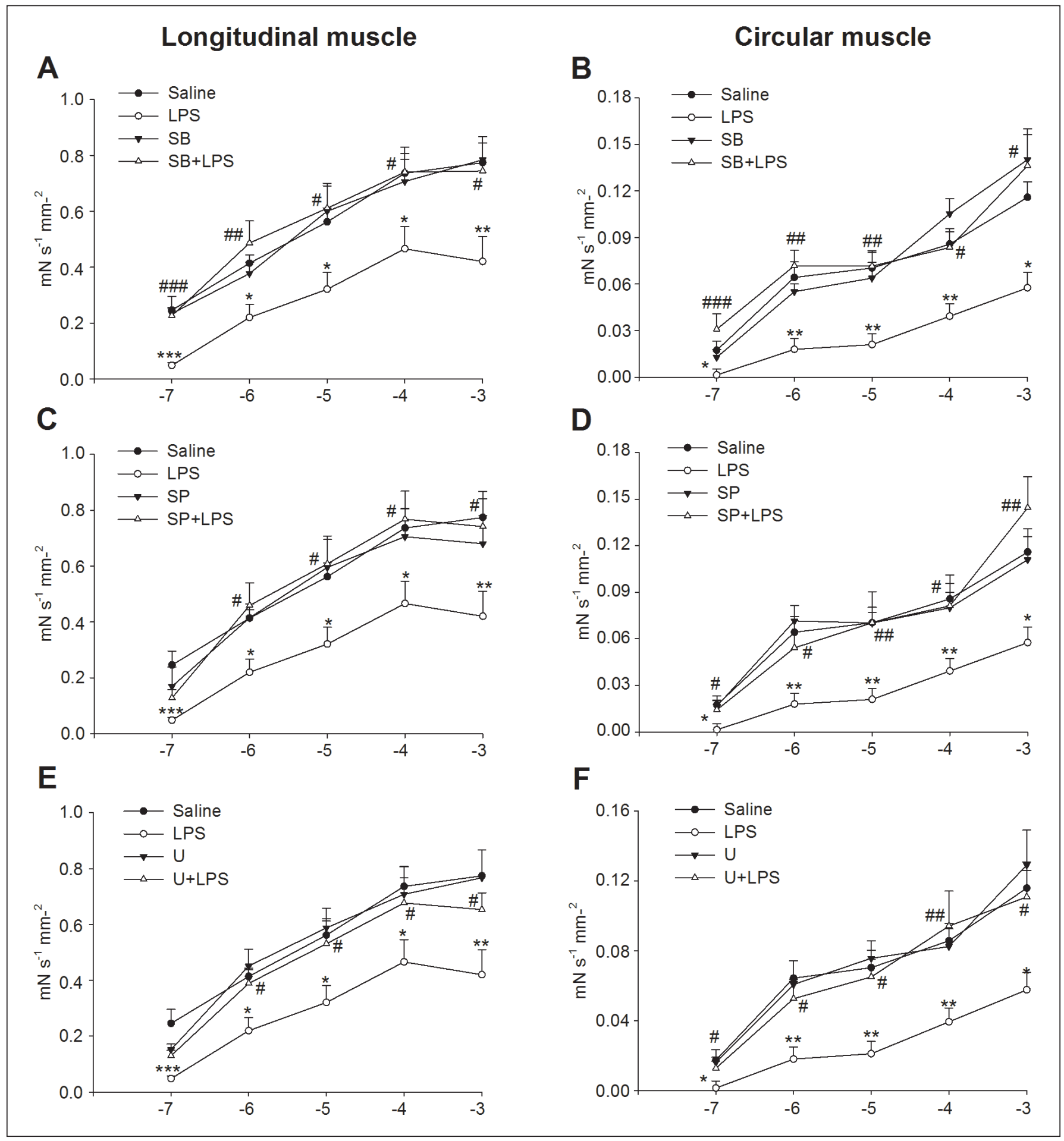

Fig. 1. Graphs representing the concentration-dependent contractile responses to acetylcholine $\left(10^{-7}\right.$ to $\left.10^{-3} \mathrm{~mol} \mathrm{~L}^{-1}\right)$ in longitudinal $(\mathrm{A}, \mathrm{C}, \mathrm{E})$ and circular $(B, D, F)$ smooth muscle of ileal segments from rabbits treated with saline (control), LPS, SB203580, SB203580 + LPS (A, B), SP600125, SP600125 + LPS (C, D), U0126 or U0126 + LPS (E, F). Each point represent mean values and vertical bars indicate S.E.M. * $p<0.05 ;{ }^{* *} p<0.01 ;{ }^{* *} p<0.001$ vs. saline; $\# p<0.05 ; \# \# p<0.01 ; \# \# \# p<0.001$ vs. LPS.

Rabbits were divided into 8 groups and were injected i.v. with either 1) saline; 2) LPS (0.2 $\left.\left.\mu \mathrm{g} \mathrm{kg}^{-1}\right) ; 3\right)$ SB203580 (30 $\left.\mu \mathrm{g} \mathrm{kg})^{-1}\right)$ 4) SB203580 + LPS; 5) SP600125 (25 $\left.\left.\mu \mathrm{g} \mathrm{kg}^{1}\right) ; 6\right)$ SP600125 + LPS; 7) U0126 (30 $\left.\mu \mathrm{g} \mathrm{kg}^{-1}\right)$ or U0126 + LPS. 


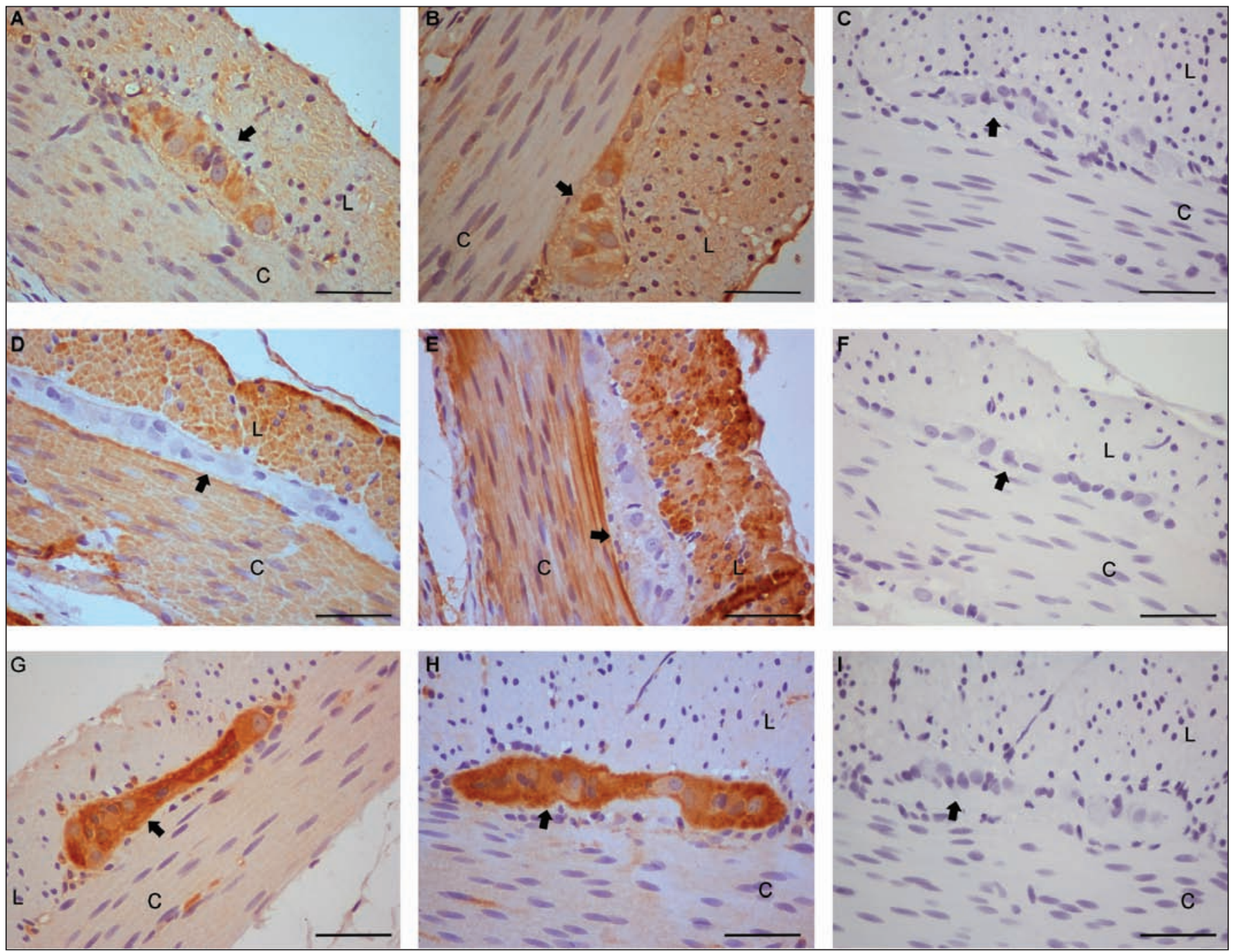

Fig. 2. Localization of p-p38, p-JNK1/2 and p-ERK1/2 in ileum of rabbits treated with LPS ( $B, E$ and $H$, respectively) or saline (A, D and G). Representative sections from 3 different animals. Bars $=50 \mu \mathrm{m}$. Magnification $\times 400$. $L$ and $C$ : longitudinal $(L)$ and circular (C) smooth muscle layers; arrow: neurons of the myenteric ganglia.

SB203580, SP600125 or U0126 were administered 15 minutes before LPS or saline. Animals were humanely killed by a blow to the head 90 minutes after LPS or saline injection.

\section{Functional studies: muscle contractility}

Pieces of rabbit ileum, were removed, washed and freed from mesenteric attachment. Isometric recordings of ileal contractility were studied in an organ bath as previously described (5). Each protocol was performed on 8 segments of ileum (4 longitudinal and 4 circular muscle) taken from the same rabbit, and repeated in three or four different animals. After the equilibration period, acetylcholine $\left(10^{-7}\right.$ to $10^{-3} \mathrm{~mol}$ $\mathrm{L}^{-1}$ ) was added to the bath for $3 \mathrm{~min}$ in a non-cumulative manner. We increased concentrations at 20 min intervals.

\section{Immunohistochemistry}

The protocol was carried out following the Vectastain $\mathrm{ABC}$ kit instructions (Vector Laboratories, CA, U.S.A.) as previously described (6). Sections were incubated at $4{ }^{\circ} \mathrm{C}$ overnight with mouse antibody anti-phosphorylated p38 (p-p38) (1:100), anti-phosphorylated JNK (p-JNK) (1:2000) or anti-phosphorylated ERK (p-ERK) (1:100) diluted in normal serum (10\%). The $p$-p38, p-JNK and p-ERK antibodies were purchased from Santa Cruz Biotechnology (Santa Cruz, CA, USA, SC7973, SC-6254 and SC-7383, respectively).

\section{Data analysis and statistics}

The acetylcholine motor responses (MR) were measured and expressed as $\mathrm{mN} \mathrm{s}^{-1} \mathrm{~mm}^{-2}$ as previously described (5). 
Results are expressed as mean \pm s.e.m. Comparisons between means were made using one-way analysis of variance (ANOVA) tests and p-values were determined using the Bonferroni's post hoc test. Differences with p-values $<0.05$ were considered statistically significant.

\section{RESULTS}

\section{Effects of SB203580, SP600125 and U0126 on LPS altered ileal contractility}

The treatment with LPS $\left(0.2 \mu \mathrm{g} \mathrm{kg}^{-1}, 90 \mathrm{~min}\right)$ decreased the acetylcholine induced response in longitudinal and circular muscle of rabbit ileum, as was previously reported in part by our laboratory (7). The treatment with SB203580 (Fig. 1A, B), SP600125 (Fig. 1C, D) or U0126 (Fig. 2E, F) blocked the effect of LPS on acetylcholine-induced contractions both in longitudinal and circular muscles. MAPKs inhibitors had no effect per se in the acetylcholine-induced contractions.

\section{Immunohistochemistry}

The expression of p-p38, p-JNK and p-ERK1/2 was found both in saline and LPS-treated rabbits. p-p38 was detected in the neurons of the myenteric plexus and smooth muscle cells of the ileum (Fig. 2A, B). p-JNK was detected in the smooth muscle cells (Fig. 2D, E). p-ERK was detected mainly in the neurons of the myenteric ganglia and slightly in the smooth muscle cells (Fig. 2G, H).

The specificity of the staining obtained was controlled by omitting the primary antibody, with a clear suppression of staining being observed in all cases (Fig. 2C, F, I).

\section{DISCUSSION}

LPS is one of the most common models used for decades to study sepsis, still one of the great challenges of medicine. Previous studies of our group show that LPS alters intestinal contractility (7). In this study we demonstrate that blockade of $\mathrm{p} 38$, JNK1/2 or ERK1/2 pathways abolished the effect of LPS on the acetylcholine-induced contractions in rabbit ileum. We have shown similar results in rabbit duodenum $(6,8,9)$ although there are some differences. The effect of LPS in the longitudinal layer is higher in the duodenum than in the ileum (58vs. $43 \%$ of reduction), while the effect of LPS is similar in the circular layer of duodenum and ileum.
In this study p-p38 was detected in the neurons of the myenteric plexus and smooth muscle cells, p-JNK in the smooth muscle cells and p-ERK mainly in the neurons of the myenteric ganglia and slightly in the smooth muscle cells of ileum. In previous studies we have described a similar localization of these MAPKs in the rabbit duodenum, although the expression of p-p38 and p-ERK1/2 in the smooth muscle of duodenum was lower than in ileum $(6,8,9)$.

In conclusion, our results suggest that $\mathrm{p} 38, \mathrm{JNK} 1 / 2$ and ERK1/2 MAPKs are involved in the effects of LPS in the ileum and, therefore, the blockade of MAPKs could be a target to avoid the intestinal motor disturbances observed in sepsis.

\section{ACKNOWLEDGMENTS}

This work was funded by the Ministerio de Ciencia y Tecnología, España (Dirección General de Investigación AGL2006-04317 and ERDF), the Gobierno de Aragón (B61/2008-2010) and the Universidad de Zaragoza (UZ2009-BIO-03). An individual grant to Sergio Gonzalo was provided by Ministerio de Educación y Ciencia, España (BES-2007-156469).

\section{REFERENCES}

1. Bauer AJ, Schwarz NT, Moore BA, Turler A, Kalff JC. Ileus in critical illness: mechanisms and management. Curr Opin Crit Care 2002;8(2): 152-7.

2. Ukleja A. Altered GI motility in critically Ill patients: current understanding of pathophysiology, clinical impact, and diagnostic approach. Nutr Clin Pract 2010;25(1):16-25.

3. Weisbrodt NW, Pressley TA, Li YF, Zembowicz MJ, Higham SC, Zembowicz A, et al. Decreased ileal muscle contractility and increased NOS II expression induced by lipopolysaccharide. Am J Physiol 1996; 271(3 Pt 1):G454-G460.

4. Kyriakis JM, Avruch J. Mammalian mitogen-activated protein kinase signal transduction pathways activated by stress and inflammation. Physiol Rev 2001;81(2):807-69.

5. Fagundes DS, Gonzalo S, Grasa L, Castro M, Arruebo MP, Plaza MA, et al. Trolox reduces the effect of ethanol on acetylcholine-induced contractions and oxidative stress in the isolated rabbit duodenum. Rev Esp Enferm Dig 2011;103(8):396-401.

6. Gonzalo S, Grasa L, Arruebo MP, Plaza MA, Murillo MD. Inhibition of p38 MAPK improves intestinal disturbances and oxidative stress induced in a rabbit endotoxemia model. Neurogastroenterol Motil 2010;22(5): 564-72,e123.

7. Rebollar E, Arruebo MP, Plaza MA, Murillo MD. Effect of lipopolysaccharide on rabbit small intestine muscle contractility in vitro: role of prostaglandins. Neurogastroenterol Motil 2002;14(6):633-42.

8. Gonzalo S, Grasa L, Arruebo MP, Plaza MA, Murillo MD. Extracellular signal-regulated kinase (ERK) is involved in LPS-induced disturbances in intestinal motility. Neurogastroenterol Motil 2011;23(2):e80-90.

9. Gonzalo S, Grasa L, Arruebo MP, Plaza MA, Murillo MD. Lipopolysaccharide-induced intestinal motility disturbances are mediated by c-Jun NH2-terminal kinases. Dig Liver Dis 2011;43(4):277-85. 